\title{
Nighttime intensivist staffing and the timing of death among ICU decedents: a retrospective cohort study
}

Lora A Reineck ${ }^{1,2}$, David J Wallace ${ }^{2,3}$, Amber E Barnato $2,4,5$ and Jeremy M Kahn 1,2,5*

\begin{abstract}
Introduction: Intensive care units (ICUs) are increasingly adopting 24-hour intensivist physician staffing. Although nighttime intensivist staffing does not consistently reduce mortality, it may affect other outcomes such as the quality of end-of-life care.
\end{abstract}

Methods: We conducted a retrospective cohort study of ICU decedents using the 2009-2010 Acute Physiology and Chronic Health Evaluation clinical information system linked to a survey of ICU staffing practices. We restricted the analysis to ICUs with high-intensity daytime staffing, in which the addition of nighttime staffing does not influence mortality. We used multivariable regression to assess the relationship between nighttime intensivist staffing and two separate outcomes potentially related to the quality of end-of-life care: time from ICU admission to death and death at night.

Results: Of 30,456 patients admitted to 27 high-intensity daytime staffed ICUs, 3,553 died in the hospital within 30 days. After adjustment for potential confounders, admission to an ICU with nighttime intensivist staffing was associated with a shorter duration between ICU admission and death (adjusted difference: -2.5 days, $95 \% \mathrm{Cl}-3.5$ to $-1.5, \mathrm{p}$-value $<0.001$ ) and a decreased odds of nighttime death (adjusted odds ratio: $0.75,95 \% \mathrm{Cl} 0.60$ to 0.94 , p-value 0.011) compared to admission to an ICU without nighttime intensivist staffing.

Conclusions: Among ICU decedents, nighttime intensivist staffing is associated with reduced time between ICU admission and death and reduced odds of nighttime death.

\section{Introduction}

Intensivist physician staffing is associated with lower mortality and decreased length of stay in the ICU $[1,2]$. This observation has led to efforts to expand the intensivist physician staffing model [3], not only during the day but also at night $[4,5]$. However, the data in support of nighttime intensivist staffing are not as robust as those for daytime intensivist staffing. Two observational before-and-after studies showed mixed results [6,7], and both a recent multicenter observational study and a single-center randomized trial concluded that nighttime intensivist staffing does not influence mortality in ICUs

\footnotetext{
* Correspondence: kahnjm@upmc.edu

'Division of Pulmonary, Allergy and Critical Care Medicine, University of Pittsburgh School of Medicine, 3459 Fifth Avenue, 628 NW, Pittsburgh, PA 15213, USA ${ }^{2}$ Department of Critical Care Medicine, Clinical Research, Investigation and Systems Modeling of Acute IIIness (CRISMA) Center, University of Pittsburgh School of Medicine, 3550 Terrace Street, Pittsburgh, PA 15261, USA Full list of author information is available at the end of the article
}

with high-intensity daytime staffing (defined as a mandatory intensivist consult or closed ICU) $[8,9]$.

Although nighttime intensivist staffing may not reduce mortality in all ICUs, extending intensivist coverage around the clock may have other benefits, including an effect on the quality of end-of-life care. The quality of end-of-life care is an important patient-centered outcome and may be impacted by nighttime intensivist staffing through earlier and more frequent conversations between physicians and surrogate decision-makers regarding prognosis and goals of care [10]. Since intensive communication leads to earlier decisions regarding life-sustaining therapy withdrawal $[11,12]$, increased communication through the addition of nighttime intensivists could result in earlier withdrawal of life-sustaining therapy and thereby alter the timing of death among ICU decedents.

The purpose of this study was to determine whether nighttime intensivist staffing is associated with the timing 
of deaths in ICU decedents. We examined two complementary elements of the timing of death: the duration between ICU admission and death, and the odds of death at night. The duration between ICU admission and death is often considered a proxy for the quality of end-of-life care, since reduced hospital and ICU stays for decedents without an increase in overall mortality represents a decrease in ineffective aggressive therapy and prolonged dying [10]. The odds of death at night may serve as a proxy for the timing of life-sustaining therapy withdrawal since the median time from terminal withdrawal of mechanical ventilation to death in the ICU is less than 1 hour [13], indicating more timely action in response to decisions to withhold life-sustaining therapy. We hypothesized that, among ICU decedents, nighttime intensivist staffing would be associated with a reduced length of time between ICU admission and death and increased odds of death at night.

\section{Materials and methods Study design}

We performed a retrospective cohort study of ICU decedents using the Acute Physiology and Chronic Health Evaluation (APACHE) clinical information system (Cerner, Kansas City, MO, USA) from 2009 through 2010. APA$\mathrm{CHE}$ collects detailed clinical, physiological, and outcome data on adult ICU patients at participating hospitals for benchmarking and quality improvement. The APACHE database has been used for numerous observational studies involving critically ill patients [14,15]. We linked these data to a 2010 survey conducted in APACHE ICUs that included questions regarding ICU organization, providers, and protocols, as previously described [8].

\section{Patients}

Patients who were 17 years of age or older and admitted to a study ICU were eligible for inclusion in the study. We excluded readmissions, patients admitted to lowintensity ICUs, those who survived to hospital discharge, and decedents whose death occurred more than 30 days after ICU admission. We limited the study to ICUs with high-intensity daytime staffing, for which nighttime intensivist staffing does not affect mortality [8], to eliminate the competing risk of death as a determinant of length of stay. We excluded patients whose death occurred more than 30 days after ICU admission to ensure that our results were not driven by long-stay outliers.

\section{Variables}

The two primary outcome variables were time from ICU admission to death and odds of death at night (defined as death occurring between 7:00 pm and 7:00 am). These variables were chosen because they may represent proxies for the quality of end-of-life care [10] and the timing of life-sustaining therapy withdrawal [13]. The primary exposure variable was presence or absence of a nighttime intensivist, defined as an intensivist attending physician who was physically present in the ICU or elsewhere in the hospital and was immediately available to manage ICU emergencies during nighttime hours [8].

Covariates were specified a priori as potential confounders between nighttime staffing and timing of death based on previous studies $[14,15]$. Patient-level covariates included age, race, sex, Acute Physiology Score (a measure of the severity of illness ranging from 0 to 252, with higher scores indicating more severe illness and a higher risk of death), the presence or absence of selected coexisting conditions (the acquired immunodeficiency syndrome, leukemia or myeloma, lymphoma, cirrhosis, liver failure, immunosuppression, and metastatic cancer), the location of the patient before admission to the ICU (emergency department, operating room, hospital floor, other hospital, or other location), the length of the hospital stay before ICU admission, the admission diagnosis, the patient's need for emergency surgery, and the patient's receipt of invasive mechanical ventilation at the time of admission [15]. We also included the teaching status of the hospital determined by the ratio of residents to beds (with a ratio of 0 indicating a nonteaching hospital, 0 to $<0.25$ a minor teaching hospital, and $\geq 0.25$ a major teaching hospital), the annualized ICU volume of admissions, and type of ICU (specialty or mixed) [16].

\section{Statistical analysis}

We compared the characteristics of hospitals with and without nighttime intensivist staffing using the MannWhitney test for continuous variables and Fisher's exact test for categorical variables. We compared patient characteristics between ICUs with and without nighttime intensivist staffing using two-sample $t$ tests for continuous variables and chi-square tests for categorical variables.

Unadjusted time from ICU admission to death was evaluated using Kaplan-Meier curves. Differences in the survivor functions between decedents admitted to ICUs with and without nighttime intensivist staffing were evaluated using the log-rank test.

We used multivariable linear regression to assess the relationship between nighttime intensivist staffing and time from ICU admission to death. We chose linear regression of untransformed length of stay over other approaches since it is a valid method for large samples regardless of distribution, including highly non-normal samples of $>500$ subjects, when the mean value is the outcome of interest [17]. We used multivariable logistic regression to assess the relationship between nighttime intensivist staffing and the odds of death at night. In all models, generalized estimating equations were used to account for clustering at the level of the ICU [18]. 
Patient race was missing in $9 \%$ of our cohort, and this variable is known to relate to the intensity of end-of-life care for hospitalized patients [19]. To account for missing race we performed multiple imputations using multinomial logistic regression [20,21], creating 10 imputed datasets. Multiple imputation was performed on the entire cohort of patients (including decedents and patients who survived to hospital discharge admitted to either low-intensity or high-intensity daytime staffed ICUs), excluding readmissions. Patient variables used for imputation included the patient-level covariates mentioned above as well as the patient's discharge disposition (dead, hospice, or alive), length of time from ICU admission to death, whether or not the patient underwent coronary artery bypass graft surgery, whether or not the patient underwent an operation, whether or not the patient received active therapy on ICU admission [22], the presence or absence of diabetes, and whether or not the patient was admitted to the ICU at nighttime $(7: 00 \mathrm{pm}$ to 7:00 am). All analyses were performed separately on each of the 10 imputed datasets, and the results were combined using Rubin's rules [23].

\section{Subgroup analyses}

We repeated our analyses in a priori selected subgroups with which we hypothesized that nighttime intensivists would interact most frequently, thereby having the largest effect on the timing of death. These subgroups included patients admitted to the ICU at night and two subgroups of patients likely to decompensate overnight due to their increased severity of illness: patients mechanically ventilated on ICU admission and patients with an Acute Physiology Score $\geq 75$ (corresponding to an approximate $25 \%$ predicted risk of in-hospital death [15]).

\section{Sensitivity analysis}

We performed a number of sensitivity analyses to assess the robustness of our findings. First, we repeated our analyses using ICU length of stay rather than time from ICU admission to death, since the time from ICU admission to death may be affected by the timing of transfer out of the ICU for patients ultimately dying on the ward. Second, we repeated the time to death analysis considering patients discharged to a hospice as decedents, since the decision to die in the hospital versus a hospice may be affected by patient and surrogate preferences. Third, we examined the effect of nighttime intensivists on the odds of death at night using a second definition of death at night $(8: 00 \mathrm{pm}$ to $8: 00 \mathrm{am})$ in order to account for the time delay between withdrawal of life-sustaining therapy and death [13].

All statistical analyses were performed using Stata 12.0 (StataCorp, College Station, TX, USA). All tests were two tailed and $P \leq 0.05$ was considered significant. This research was approved by the Institutional Review Board of the University of Pittsburgh. A waiver for individual informed consent was provided due to the minimal risk for participants.

\section{Results}

The initial cohort included 64,752 admissions to 49 ICUs in 25 hospitals. A total of 3,553 decedents admitted to 27 high-intensity daytime staffed ICUs in 15 hospitals met the study inclusion criteria (Figure 1). As previously reported, the odds ratio for in-hospital death in this cohort's high-intensity daytime staffed ICUs with nighttime staffing compared with the ICUs without nighttime intensivist staffing was 1.08 (95\% confidence interval, 0.63 to $1.84, P=0.78$ ) [8]. Six ICUs with nighttime intensivists contributed data on 863 decedents, and 21 ICUs without nighttime intensivists contributed data on 2,690 decedents. Characteristics of the 27 ICUs in the study are shown in Table 1. The ICUs without nighttime intensivists more commonly had a full-time ICU director and were located within a large hospital. Other characteristics of the ICUs were similar.

Characteristics of the 3,553 decedents in the study are shown in Table 2. Decedents admitted to ICUs with nighttime intensivists had a shorter time between ICU admission and death, a shorter ICU length of stay, and fewer nighttime deaths compared with those admitted to ICUs without nighttime intensivists. A Kaplan-Meier survival curve demonstrated that the unadjusted time from ICU admission to death was significantly shorter in ICUs with nighttime intensivists compared with those without through 30 days of follow-up (Figure 2, log-rank $P<0.001)$.

In the multivariable analysis, we found that adjusted length of time from ICU admission to death was significantly shorter among decedents admitted to ICUs with nighttime intensivists compared with those without (Table 3). This was also true for each of the subgroups analyzed. The adjusted odds of death at night was significantly lower among decedents admitted to ICUs with nighttime intensivists compared with those admitted to ICUs without nighttime intensivists (Table 4). The difference in the odds of death at night was not significant in any of the subgroups, although the odds ratios were similar to those of the full cohort. The sensitivity analyses yielded similar results (Additional file 1: Table S1).

\section{Discussion}

In a retrospective cohort study of decedents admitted to high-intensity daytime staffed ICUs, the presence of a nighttime intensivist was associated with a decrease in time from ICU admission to death by an average of 2.5 days. This finding suggests that the addition of nighttime intensivists to high-intensity daytime staffed ICUs may 


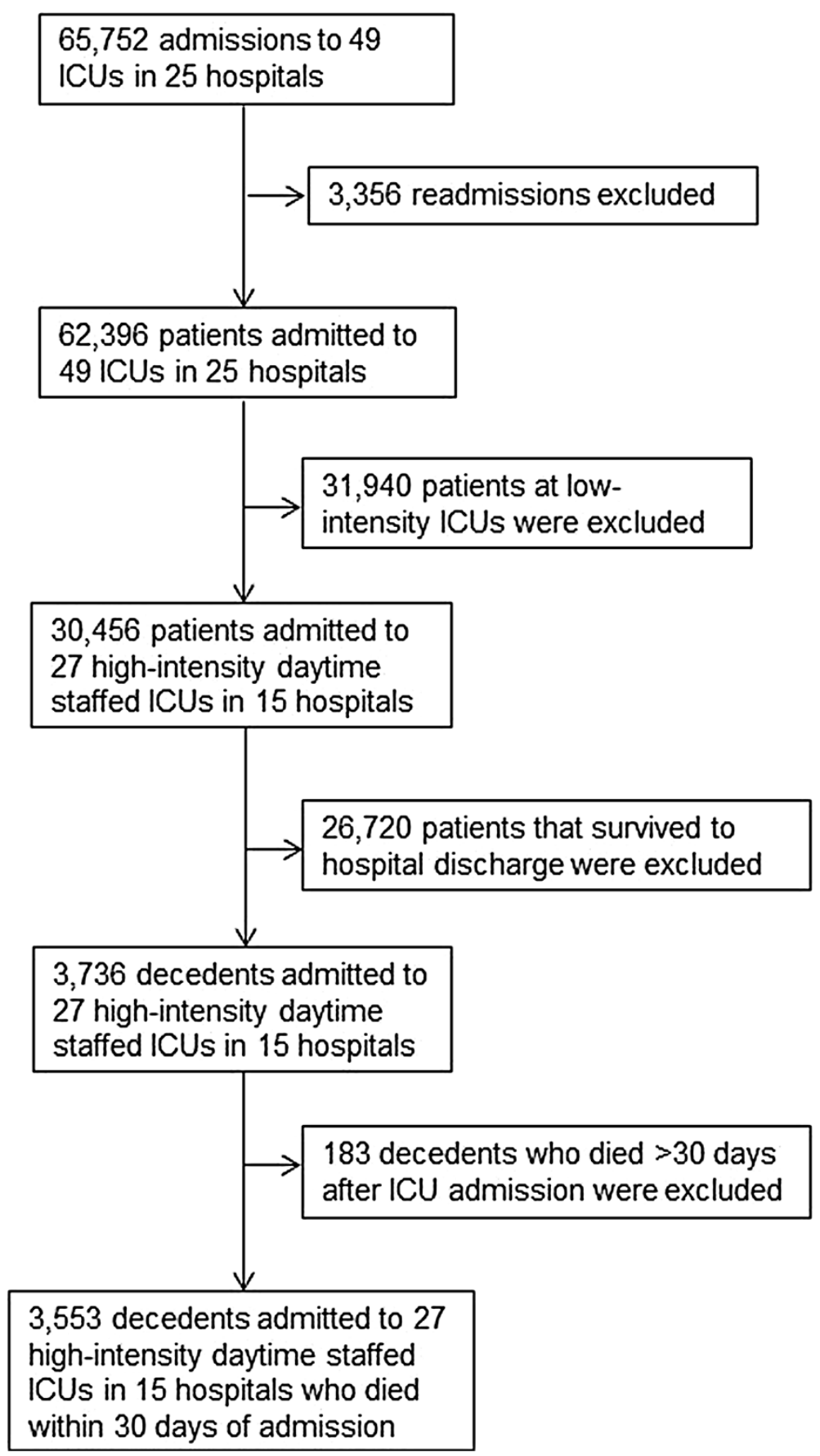

Figure 1 Number of decedents, ICUs, and hospitals in the study.

improve the quality of end-of-life care for ICU decedents. A possible mechanism may be earlier and more frequent conversations between intensivists and surrogates regarding prognoses and goals of care, leading to earlier withdrawal of life-sustaining treatment. Nighttime intensivist staffing may improve both the timing of communication with surrogates and surrogates' access to physicians, which research suggests are two aspects of end-of-life care in need of improvement [24]. As a result, decisions to limit life-sustaining therapy may occur earlier in the ICU course [11,12], resulting in earlier transition to palliative care for patients and increased family and clinician satisfaction [25].

Contrary to our hypothesis, we found that the addition of a nighttime intensivist to high-intensity daytime staffed ICUs was associated with decreased odds of death at 
Table 1 Characteristics of high-intensity daytime staffed ICUs

\begin{tabular}{lccc}
\hline Characteristic & $\begin{array}{c}\text { Nighttime } \\
\text { intensivists } \\
(\boldsymbol{n}=\mathbf{6})\end{array}$ & $\begin{array}{c}\text { No nighttime } \\
\text { intensivists } \\
(\boldsymbol{n}=\mathbf{2 1})\end{array}$ & $\boldsymbol{P}$ value \\
\hline $\begin{array}{l}\text { Annualized ICU } \\
\text { admissions }\end{array}$ & $517(418$ to 603) & $770(514$ to 979) & 0.24 \\
ICU type & $3(50)$ & $11(52)$ & $>0.99$ \\
$\quad$ Mixed & $3(50)$ & $10(48)$ & \\
$\quad$ Specialty & $2(33)$ & $20(95)$ & 0.004 \\
$\begin{array}{l}\text { Full-time physician } \\
\text { ICU director }\end{array}$ & $5(83)$ & $21(100)$ & 0.22 \\
Routine participation \\
of medical students, \\
residents, or other \\
physician trainees
\end{tabular}

Data presented as median (interquartile range) or $\mathrm{n}(\%)$.

${ }^{a}$ Teaching status categorized by resident-to-bed ratio (nonteaching, 0 ; minor teaching, $>0$ to $<0.25$; major teaching, $\geq 0.25$ )

night. Although nighttime intensivist staffing may lead to earlier withdrawal of life-sustaining treatment, this finding suggests that it is unlikely to be occurring more frequently at night. Instead, nighttime intensivists may have discussions with surrogates preparing them for withdrawal of life-sustaining treatment by the daytime team. Additionally, the decreased odds of death at night may indicate that nighttime intensivists are better able to manage decompensating patients overnight than non-intensivists, forestalling death in the short term. Fewer deaths at night may allow more family members to be present at the time of life-sustaining treatment withdrawal and patient death, further supporting the notion that nighttime intensivists may improve the quality of end-of-life care for ICU decedents [26].

Our findings have implications for the many ICUs that have already implemented nighttime intensivist staffing or those that are considering implementing nighttime
Table 2 Characteristics of decedents in high-intensity daytime staffed ICUs ${ }^{\mathrm{a}}$

\begin{tabular}{lccc}
\hline Characteristic & $\begin{array}{c}\text { ICUs with } \\
\text { nighttime } \\
\text { intensivists } \\
(\boldsymbol{n}=\mathbf{8 6 3})\end{array}$ & $\begin{array}{c}\text { ICUs without } \\
\text { nighttime } \\
\text { intensivists } \\
(\boldsymbol{n}=\mathbf{2 , 6 9 0 )}\end{array}$ & \\
\hline Age (years) & $71(58$ to 80$)$ & $68(56$ to 79$)$ & 0.001 \\
Female sex & $393(45.5)$ & $1229(45.7)$ & 0.94 \\
Race & $719(83.3)$ & $2009(74.7)$ & \\
White & $29(3.4)$ & $301(11.2)$ & \\
Black & $113(13.1)$ & $70(2.6)$ & \\
Other & $2(0.2)$ & $310(11.5)$ & \\
Data missing & & & $<0.001$ \\
Admission source & $331(38.4)$ & $1145(42.6)$ & \\
Emergency department & $77(8.9)$ & $418(15.5)$ & \\
Operating room & $221(25.6)$ & $769(28.6)$ & \\
Medical or surgical ward & $223(25.8)$ & $286(10.6)$ & \\
Transfer & $11(1.3)$ & $72(2.7)$ & \\
Other & & & $<0.001$ \\
Reason for ICU admission & &
\end{tabular}

Reason for ICU admission

Surgery
Cardiac disorder
Respiratory disorder
General medical disorder
Sepsis
Trauma
Neurosurgery
Cardiac arrest
Other

$64(7.4) \quad 333(12.4)$

$102(11.8) \quad 233(8.7)$

$199(23.1) \quad 411(15.3)$

$49(5.7) \quad 145(5.4)$

$92(10.7) \quad 511(19.0)$

$23(2.7) \quad 187(7.0)$

$11(1.3) \quad 44(1.6)$

$122(14.1) \quad 291(10.8)$

$201(23.3) \quad 535(19.9)$

Acute physiology score

63 (45 to 85$) \quad 71(51$ to 97$) \quad<0.001$

Active treatment on day of ICU admission ${ }^{\mathrm{b}}$

Mechanical ventilation on day of ICU admission

Emergency surgery

$749(86.8) \quad 2397(89.1) \quad 0.06$

Length of hospital stay before ICU admission (days)

ICU length of stay (days) 2.7 (1.1 to 6.0$) \quad 3.0(1.3$ to 6.8$) \quad<0.001$

Length of time from ICU admission to death (days)

Deaths at night ${ }^{\mathrm{C}}$

$309(35.8)$

$1130(42.0)$

0.001

Data presented as median (interquartile range) or $\mathrm{n}(\%)$.

apatient characteristics in this table are those that were observed, not imputed or adjusted.

${ }^{\mathrm{b}}$ Active treatment was defined as any of 33 active life-supporting intensive care treatments [21]

${ }^{c}$ Night defined as 7:00 pm to 7:00 am.

staffing. A relationship between nighttime intensivists and the quality of end-of-life care for ICU decedents may provide a rationale for the continuation or implementation of the nighttime intensivist ICU staffing model, even 


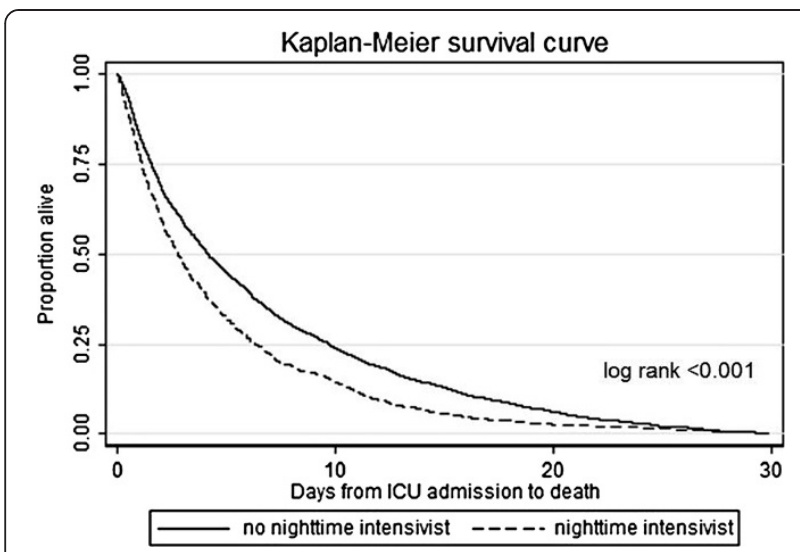

Figure 2 Kaplan-Meier curve for time from ICU admission to death. Time from ICU admission to death (days) for decedents admitted to ICUs with nighttime intensivists compared with ICUs without nighttime intensivists.

in the absence of a mortality benefit. Additionally, the reduction in time between ICU admission and death represents decreased resource utilization.

Our study has several limitations. The hospitals in this study were a self-selected group of hospitals participating in the APACHE clinical information system rather than a random sample. Compared with other hospitals in the United States, APACHE hospitals are generally larger and more likely to be affiliated with an academic institution. Therefore it is not known whether the findings in this study would be applicable to all other high-intensity daytime staffed ICUs. Second, we acknowledge that confounding by unmeasured ICU characteristics may have affected our results. For example, ICUs with nighttime intensivist staffing may have different norms regarding the use of time-limited trials of life-sustaining treatment for patients than ICUs without nighttime intensivist staffing; this could be an alternate explanation for the reduced time between ICU admission and death. Third, we did not directly measure the quality of end-oflife care; we used a proxy. We acknowledge that there is

Table 3 Adjusted time from ICU admission to death for ICUs with versus without nighttime intensivists

\begin{tabular}{lccc}
\hline Cohort & $\boldsymbol{N}$ & Days $(\mathbf{9 5 \%} \mathrm{Cl})$ & $\boldsymbol{P}$ value \\
\hline $\begin{array}{l}\text { Complete cohort } \\
\text { Subgroups }\end{array}$ & 3,553 & $-2.5(-3.5$ to -1.5$)$ & $<0.001$ \\
$\begin{array}{l}\text { Patients admitted to the } \\
\text { ICU at night }\end{array}$ & 1,647 & $-3.0(-4.0$ to -1.9$)$ & $<0.001$ \\
$\begin{array}{l}\text { Patients mechanically } \\
\text { ventilated on ICU admission }\end{array}$ & 2,671 & $-2.1(-2.9$ to -1.2$)$ & $<0.001$ \\
$\begin{array}{l}\text { Patients with Acute Physiology } \\
\text { Score } \geq 75\end{array}$ & 1,551 & $-1.6(-2.5$ to -0.7$)$ & 0.001 \\
\hline
\end{tabular}

$\mathrm{Cl}$, confidence interval.
Table 4 Adjusted odds ratio for death at night in ICUs with versus without nighttime intensivists

\begin{tabular}{lccc}
\hline Cohort & $\boldsymbol{N}$ & Odds ratio $(\mathbf{9 5 \%} \mathrm{Cl})$ & $\boldsymbol{P}$ value \\
\hline $\begin{array}{l}\text { Complete cohort } \\
\text { Subgroups }\end{array}$ & 3,553 & $0.75(0.60$ to 0.94$)$ & 0.01 \\
$\begin{array}{l}\text { Patients admitted to the } \\
\text { ICU at night }\end{array}$ & 1,647 & $0.76(0.56$ to 1.03$)$ & 0.08 \\
$\begin{array}{l}\text { Patients mechanically } \\
\text { ventilated on ICU admission }\end{array}$ & 2,671 & $0.81(0.62$ to 1.05$)$ & 0.11 \\
$\begin{array}{l}\text { Patients with Acute Physiology } \\
\text { Score } \geq 75\end{array}$ & 1,551 & $0.95(0.68$ to 1.33$)$ & 0.76 \\
\hline $\begin{array}{l}\text { Cl, confidence interval. } \\
\end{array}$ & & & \\
\hline
\end{tabular}

$\mathrm{Cl}$, confidence interval.

limited evidence regarding the relationship between timing of death and the quality of end-of-life care, and that a shorter duration between ICU admission and death may not be associated with higher family satisfaction in all cases [27]. Future work should directly examine the relationship between nighttime staffing and the quality of end-of-life care. Finally, we did not assess the behavior of nighttime intensivists in terms of their communication with patients and families, the timing of patient or family decisions regarding life-sustaining treatment, or whether or not death occurred following a decision to withdraw life-sustaining treatment. These variables were not available in our data. Future work should take a more granular approach and examine how nighttime intensivists and surrogates interact and when treatment decisions occur. This would allow a better understanding of the true mechanisms of our findings and help uncover ways to optimize the role of nighttime intensivists in the dying process.

\section{Conclusions}

Among ICU decedents, the addition of nighttime intensivist staffing to high-intensity daytime staffing is associated with a reduced time between ICU admission and death and reduced odds of death at night.

\section{Key messages}

- Among decedents admitted to high-intensity daytime staffed ICUs, the addition of a nighttime intensivist was associated with a reduced time between ICU admission and death.

- Among decedents admitted to high-intensity daytime staffed ICUs, the addition of a nighttime intensivist was associated with reduced odds of death at night.

- The addition of nighttime intensivist staffing to high-intensity daytime staffed ICUs may improve the quality of end-of-life care. 


\section{Additional file}

Additional file 1: Table S1. presenting results of sensitivity analyses comparing ICUs with versus without nighttime intensivists.

\section{Abbreviation}

APACHE: Acute physiology and chronic health evaluation.

\section{Competing interests}

The authors declare that they have no competing interests.

\section{Authors' contributions}

LAR contributed to the study design, performed analysis and interpretation of the data, and drafted the manuscript. DJW contributed to the analysis and interpretation of the data and revision of the manuscript. AEB contributed to the analysis and interpretation of the data and revision of the manuscript. JMK conceived the study and study design, assisted in the analysis and interpretation of the data, and revised the manuscript. All authors read and approved the final manuscript.

\section{Authors' information}

LAR is a pulmonary and critical care fellow at the University of Pittsburgh. DJW is an Assistant Professor of Critical Care Medicine and Emergency Medicine at the University of Pittsburgh. AEB is an Associate Professor of Medicine, Clinical and Translational Science, and Health Policy \& Management at the University of Pittsburgh. JMK is an Associate Professor of Critical Care, Medicine, and Health Policy \& Management at the University of Pittsburgh.

\section{Acknowledgements}

The authors acknowledge the following funding sources: National Institutes of Health (NIH) T32HL7563 (to LAR), NIH K12HL109068 (to DJW), and NIH K23HL082650 and NIH R01HL096651 (to JMK). The funding bodies had no role in the study design; the collection, analysis, and interpretation of data; writing of the manuscript; or the decision to submit the manuscript for publication.

\section{Author details}

${ }^{1}$ Division of Pulmonary, Allergy and Critical Care Medicine, University of Pittsburgh School of Medicine, 3459 Fifth Avenue, 628 NW, Pittsburgh, PA 15213, USA ${ }^{2}$ Department of Critical Care Medicine, Clinical Research, Investigation and Systems Modeling of Acute IIIness (CRISMA) Center, University of Pittsburgh School of Medicine, 3550 Terrace Street, Pittsburgh, PA 15261, USA. ${ }^{3}$ Department of Emergency Medicine, University of Pittsburgh School of Medicine, Suite 10028 Forbes Tower, Pittsburgh, PA 15260, USA. ${ }^{4}$ Division of General Internal Medicine, University of Pittsburgh School of Medicine, UPMC Montefiore Hospital, Suite W933 200 Lothrop Street, Pittsburgh, PA 15213, USA. ${ }^{5}$ Department of Health Policy and Management, University of Pittsburgh Graduate School of Public Health, 130 De Soto Street, Pittsburgh, PA 15261, USA.

Received: 15 May 2013 Accepted: 23 July 2013

Published: 3 October 2013

\section{References}

1. Pronovost PJ, Angus DC, Dorman T, Robinson KA, Dremsizov TT, Young TL: Physician staffing patterns and clinical outcomes in critically ill patients: a systematic review. JAMA 2002, 288:2151-2162.

2. Dimick JB, Pronovost PJ, Heitmiller RF, Lipsett PA: Intensive care unit physician staffing is associated with decreased length of stay, hospital cost, and complications after esophageal resection. Crit Care Med 2001, 29:753-758.

3. Factsheet: ICU Physician Staffing. [http://www.leapfroggroup.org/media/file/ Leapfrog-ICU_Physician_Staffing_Fact_Sheet.pdf]

4. Burnham EL, Moss M, Geraci MW: The case for $24 / 7$ in-house intensivist coverage. Am J Respir Crit Care Med 2010, 181:1159-1160.

5. Cartin-Ceba R, Bajwa EK: 24-hour on-site intensivist in the intensive care unit: yes. Am J Respir Crit Care Med 2010, 181:1279-1280.

6. Blunt MC, Burchett KR: Out-of-hours consultant cover and case-mixadjusted mortality in intensive care. Lancet 2000, 356:735-736.
7. Gajic O, Afessa B, Hanson AC, Krpata T, Yilmaz M, Mohamed SF, Rabatin JT, Evenson LK, Aksamit TR, Peters SG, Hubmayr RD, Wylam ME: Effect of 24-hour mandatory versus on-demand critical care specialist presence on quality of care and family and provider satisfaction in the intensive care unit of a teaching hospital. Crit Care Med 2008, 36:36-44.

8. Wallace DJ, Angus DC, Barnato AE, Kramer AA, Kahn JM: Nighttime intensivist staffing and mortality among critically ill patients. N Engl J Med 2012, 366:2093-2101.

9. Kerlin MP, Small DS, Cooney E, Fuchs BD, Bellini LM, Mikkelsen ME, Schweickert WD, Bakhru RN, Gabler NB, Harhay MO, Hansen-Flaschen J, Halpern SD: A randomized trial of nighttime physician staffing in an intensive care unit. N Engl J Med 2013, 368:2201-2209.

10. Engelberg RA: Measuring the quality of dying and death: methodological considerations and recent findings. Curr Opin Crit Care 2006, 12:381-387.

11. Quenot JP, Rigaud JP, Prin S, Barbar S, Pavon A, Hamet M, Jacquiot N, Blettery B, Herve C, Charles PE, Moutel G: Impact of an intensive communication strategy on end-of-life practices in the intensive care unit. Intensive Care Med 2012, 38:145-152.

12. Lilly CM, De Meo DL, Sonna LA, Haley KJ, Massaro AF, Wallace RF, Cody S: An intensive communication intervention for the critically ill. Am J Med 2000, 109:469-475.

13. Cooke CR, Hotchkin DL, Engelberg RA, Rubinson L, Curtis JR: Predictors of time to death after terminal withdrawal of mechanical ventilation in the ICU. Chest 2010, 138:289-297.

14. Kahn JM, Goss CH, Heagerty PJ, Kramer AA, O'Brien CR, Rubenfeld GD: Hospital volume and the outcomes of mechanical ventilation. N Engl J Med 2006, 355:41-50.

15. Zimmerman JE, Kramer AA, McNair DS, Malila FM: Acute Physiology and Chronic Health Evaluation (APACHE) IV: hospital mortality assessment for today's critically ill patients. Crit Care Med 2006, 34:1297-1310.

16. Lott JP, Iwashyna TJ, Christie JD, Asch DA, Kramer AA, Kahn JM: Critical illness outcomes in specialty versus general intensive care units. Am J Respir Crit Care Med 2009, 179:676-683.

17. Lumley T, Diehr P, Emerson S, Chen L: The importance of the normality assumption in large public health data sets. Annu Rev Public Health 2002, 23:151-169.

18. Zeger SL, Liang KY: Longitudinal data analysis for discrete and continuous outcomes. Biometrics 1986, 42:121-130.

19. Barnato AE, Chang CC, Saynina O, Garber AM: Influence of race on inpatient treatment intensity at the end of life. J Gen Intern Med 2007, 22:338-345

20. Raghunathan TE, Lepkowski JM, Van Hoewyk J, Solenberger P: A multivariate technique for multiply imputing missing values using a sequence of regression models. Survey Methodology 2001, 27:85-95.

21. van Buuren S: Multiple imputation of discrete and continuous data by fully conditional specification. Stat Methods Med Res 2007, 16:219-242.

22. Zimmerman JE, Kramer AA: A model for identifying patients who may not need intensive care unit admission. J Crit Care 2010, 25:205-213.

23. Rubin DB: Multiple Imputation for Non-response in Surveys. New York: John Wiley \& Sons; 1987.

24. Hanson LC, Danis M, Garrett J: What is wrong with end-of-life care? Opinions of bereaved family members. J Am Geriatr Soc 1997, 45:1339-1344.

25. Boyle DK, Miller PA, Forbes-Thompson SA: Communication and end-of-life care in the intensive care unit: patient, family, and clinician outcomes. Crit Care Nurs Q 2005, 28:302-316.

26. Glavan BJ, Engelberg RA, Downey L, Curtis JR: Using the medical record to evaluate the quality of end-of-life care in the intensive care unit. Crit Care Med 2008, 36:1138-1146.

27. Gerstel E, Engelberg RA, Koepsell T, Curtis JR: Duration of withdrawal of life support in the intensive care unit and association with family satisfaction. Am J Respir Crit Care Med 2008, 178:798-804.

\section{doi:10.1186/cc13033}

Cite this article as: Reineck et al:: Nighttime intensivist staffing and the timing of death among ICU decedents: a retrospective cohort study. Critical Care 2013 17:R216. 